\section{APROXIMACIÓN TEÓRICA Y METALOGRÁFICA A LA REDUCCIÓN DE HIERRO EN LA PREHISTORIA PARTIENDO DEL TRABAJO EXPERIMENTAL (1)}

\author{
APPROACH TO IRON REDUCTION \\ IN PREHISTORY THROUGH \\ THEORETICAL, METALOGRAPHICAL \\ AND EXPERIMENTAL WORK
}

\author{
FRANCISCO JAVIER SARABIA \\ HERRERO (*)
}

\section{RESUMEN}

El presente artículo describe una experiencia de reducción de hierro realizada en Dinamarca en una reproducción de horno vertical basada en hallazgos datados en la II Edad del Hierro danesa. Igualmente, se realiza un encuadre teórico de dicha experiencia, tanto desde el punto de vista químico como del arqueológico, y se inluye un examen metalográfico del hierro obtenido cara

(1) Quiero expresar mi agradecimiento a la Dirección General de Investigación Científica y Técnica del M.E.C. Igualmente, agradezco al profesor Joorgen Lund de la Universidad de Aarhus (Dinamarca) su apoyo en los trabajos realizados en el Departamento de Arqueología Prehistórica de Moesgaard (Aarhus), así como a D. Jesús Pérez Cabellos (Laboratorio de Química Inorgánica y Análisis E.T.S.I.I. de la Universidad de Valladolid) su gran ayuda en la realización e interpretación de las metalografías presentadas en este artículo.

$\left.{ }^{*}\right)$ Becario (B.F.P.I.) del Departamento de Prehistoria, Arqueología, Antropología Social y Ciencias y Técnicas Historiográficas de la Universidad de Valladolid. Plaza de la Universidad, s/n. 47002 Valladolid

$\mathrm{El}$ artículo fue remitido en su versión final el 3-II-93. a su posible utilización en la investigación de piezas históricas de hierro.

\begin{abstract}
The starting point of this paper is the description of an experimental iron reduction developed using a reconstruction of a shaft kiln based upon finds dated to the Danish Iron Age. A chemical and archaeological perspective on the experience is given. A metalographical study of the resulting metal was also made, both to check the iron obtained and to get pictures that may be useful in the future investigation of historical pieces of iron.
\end{abstract}

Palabras clave: Arqueometalurgia. Hierro. Reducción. Química. Experimental. Metalografía. Prehistoria Danesa.

Key words: Archaeometalurgy. Iron. Reduction. Chemistry. Experimental. Metalography. Danish Prehistory. 


\section{INTRODUCCIÓN}

La reducción del hierro es uno de los aspectos al que se ha prestado más atención dentro del campo de la arqueometalurgia. Se ha logrado alcanzar una serie de avances muy claros en el conocimiento de las técnicas de reducción en la Antigüedad, lo que no quiere decir, en absoluto, que este conocimiento sea suficiente ni esté exento de controversias.

Una de las vías más interesantes seguidas por la investigación ha sido la de la experimentación. En este trabajo se pretende describir una de estas experiencias, en la que se tuvo la oportunidad de participar durante una visita de trabajo de seis meses al Departamento de Prehistoria de la Universidad de Aarhus (Dinamarca), centro que cuenta con una larga tradición en la realización de trabajos de arqueología experimental. Consistió en una reducción de hierro empleando una reproducción de horno vertical (también llamados a veces, por traducción literal del inglés, horno de chimenea), realizada en la primavera de 1990 en el Museo de Moesgaard (Aarhus, Dinamarca).

Este experimento formaba parte de un proyecto más amplio dirigido por el profesor Joorgen Lund del Departamento de Prehistoria de la Universidad de Aarhus, quien habrá de publicar todos los datos cuantitativos relativos a esta práctica. Por mi parte, una vez en España, realicé un estudio metalográfico del metal obtenido en el Laboratorio de Química Inorgánica de la Escuela Técnica Superior de Ingenieros Industriales de Valladolid bajo la dirección del Dr. Jesús Martín Gil. Se pretende ahora describir dicha experiencia enmarcándola en otros dos aspectos básicos: el teórico y el cultural. Con todo ello, se quiere dar un encuadre que pueda servir de aproximación a la consecución de hierro metal en la Prehistoria, encuadre que, por su carácter técnico, puede ser válido no sólo para el Norte de Europa sino también para la Península Ibérica y, por tanto, puede complementar los todavía escasos estudios del hierro en España realizados desde el punto de vista de la arqueometría, como los de Keesmann y Niemayer (1989), Madroñero de la Cal (1989), Hernández y Murillo (1985), los recientemente publicados por la Universidad de Murcia (Arana et alii, 1993), o como el que están realizando desde Salamanca el Dr. Angel Esparza Arroyo y D. Javier Larrazábal Galarza en el N-O del Valle del Duero.

El artículo ha sido estructurado en tres partes (teórica, experimental y metalográfica) incluyéndose una serie de aclaraciones relativas a los fundamentos químicos de la reducción y al período histórico al que corresponden los modelos empleados para la reconstrucción.

\section{PRINCIPIOS TEÓRICOS DE LA REDUCCIÓN}

Se puede definir la reducción de diferentes formas de acuerdo con el punto de vista que se tome. Propiamente, supone la ganancia de electrones por parte de una especie química (mientras que la oxidación sería la pérdida). Puesto que en todos los compuestos de interés metalúrgico los metales se encuentran en estados de oxidación positivos, el proceso último a realizar es siempre una reducción. Pero también puede decirse que la reducción es el proceso que implica la pérdida de moléculas de oxígeno por parte de compuestos que contengan átomos metálicos, o bien, simplemente, que es aquél proceso cuyo resultado es la consecución de metal puro a partir de un mineral.

Constituye, con la forja, una de las etapas fundamentales en que se puede estructurar la realización de manufacturas de hierro durante el Mundo Antiguo. Una primera aclaración importante es que ambos procesos implican la producción de escoria (y, por tanto, su presencia en un yacimiento no basta para poder concluir que en él se realizasen procesos de reducción). Las escorias primitivas suelen ser predominantemente fayalitas $\left(2 \mathrm{FeO} . \mathrm{SiO}_{2}\right.$, un silicato de hierro) con cantidades variables de wüstita $(\mathrm{FeO})$ y una matriz vítrea, los tres con un eutéctico a $1170^{\circ} \mathrm{C}$, que puede absorber pequeñas cantidades de elementos como $\mathrm{MnO}, \mathrm{CaO}, \mathrm{Al}_{2} \mathrm{O}_{3}$, etc., que no modifican el punto más bajo de fusión en más de $\pm 50^{\circ} \mathrm{C}$ (Tylecote, $1986: 129$ y 130). El interés de las escorias es grande, ya que mientras la composición de los hierros en bruto suele ser bastante parecida independientemente del tipo de horno, la de las escorias sí que está más influída por el horno que por la composición original del mineral (Rostoker y Dvorak, 1990: 153).

Otro aspecto que no debe olvidarse es que la reducción no puede ser identificada con fusión. La primera es un fenómeno químico que im-

T. P., 51, nº 1, 1994 
plica un cambio en la composición molecular, mientras que la segunda es un mero cambio de estado sin variación en la composición.

En el caso del hierro, nunca llegó a fundirse en el Viejo Mundo como muy pronto hasta la Edad Moderna (en general, hasta la Revolución Industrial). No es el único caso de reducción en sólido que se dio en la Prehistoria: algunos autores (Budd et alii, 1992: 5) creen que el cobre también fue reducido en algunos momentos sin llegar a la licuación.

El hierro puede ser reducido a unos $800^{\circ} \mathrm{C}$, muy por debajo de su punto de fusión (a $1536^{\circ} \mathrm{C}$ ). En estas condiciones, uno de los problemas principales es la ganga del mineral, que debe ser eliminada por medio de creación y licuación de escoria a altas temperaturas (unos $1100^{\circ} \mathrm{C}$ ).

Veamos este problema. Dado que la reducción tiene lugar por debajo del punto de fusión, el lingote de hierro bruto que se obtiene al final se produce por crecimiento de cristales de hierro en estado sólido. Por ello es fundamental ir eliminando todo aquello que pueda impedir la formación de un único bloque de hierro (es decir, la ganga) y es también por este motivo que el resultado es una especie de esponja con inclusiones de escoria. Algunos autores (Tylecote, 1986: 129) consideran que en los hornos más antiguos es gracias al hierro aportado por el propio mineral que la escoria bajaba su punto de fusión (sílica y alúmina, sus componentes principales, tienen una fusión en torno a los $1700^{\circ} \mathrm{C}$ ) quedando por debajo de los $1200^{\circ} \mathrm{C}$. Esto implica que gran parte del hierro pasaba a la escoria y, por tanto, según Tylecote, se trata de procedimientos muy ineficaces desde un punto de vista moderno, explicándose así que en el s. XVII y en adelante hubiera un comercio de escorias antiguas como mena: en ese momento, los hornos podían alcanzar temperaturas más altas y usar grandes cantidades de cal como fundente en vez del hierro, resultando una escoria con un punto de fusión en torno a los $1400^{\circ} \mathrm{C}$.

Sin embargo, las suposiciones de Tylecote pueden dar lugar a confusión si se consideran aisladas. Para intentar entender la naturaleza de los procesos que estamos comentando necesitamos considerar siquiera someramente una serie de principios de la Termodinámica Física.

Es fundamental tener presentes los diagramas de energía libre o de Ellingham (Fig. 1). Una buena referencia a ellos puede encontrarse en Manku (1983: 406 a 412), al que nos ceñiremos fundamentalmente, o bien en Gilchrist (1989: 147 a 150).

Los diagramas de Ellingham representan gráficamente las energías libres tipo de formación de óxidos metálicos a diferentes temperaturas. En ellos se puede ver que, en general, la estabilidad termodinámica de los óxidos disminuye al aumentar la temperatura, al tiempo que se puede obtener una secuencia de estabilidad de los óxidos metálicos trazando una línea vertical a cualquier temperatura: un metal que forme un óxido metálico más estable aparecerá más abajo en el diagrama y será un agente reductor potencial para un óxido menos estable. De todas formas, el oro y otros metales preciosos pueden existir nativos, y sus óxidos pueden ser reducidos sin que intervenga otro elemento simplemente por calentamiento a las temperaturas en que sus energías libres de formación se hacen iguales a cero.

Para entender la función de los agentes reductores hay que recordar que en una reacción redox (es decir, de oxidación-reducción) mientras un elemento se libera de moléculas de oxígeno y se reduce (en el caso que nos ocupa, el hierro), otro (generalmente el carbono) experimenta el fenómeno contrario y se oxida.

El carbono es el agente reductor más importante. Es capaz de reducir cualquiera de los metales de interés en Arqueología con tal de que se proporcione una temperatura suficientemente alta. También es necesario que éstos se encuentren formando óxidos y no sulfuros, ya que la entalpía de formación del disulfuro de carbono es endotérmica (mientras que los sulfuros metálicos son sólidos muy estables) lo que hace del carbono un agente muy poco adecuado para reducir sulfuros. De todas formas, el oxígeno (que forma el $50 \%$ de la corteza terrestre) se encuentra naturalmente asociado con muchos metales y es fácil obtener óxidos metálicos de las menas naturales mediante un simple proceso de tostado o calcinación.

El carbono forma dos óxidos: el monóxido de carbono ( $\mathrm{CO}$ ), cuya formación se va haciendo más favorable con el aumento de la temperatura (lo que queda representado en el diagrama de Ellingham por una línea de pendiente negativa), y el dióxido de carbono $\left(\mathrm{CO}_{2}\right)$, más estable a bajas (con una línea de pendiente casi nula). De ellos, el CO es el agente reductor más activo por debajo de los $760^{\circ} \mathrm{C}$, mientras que a 


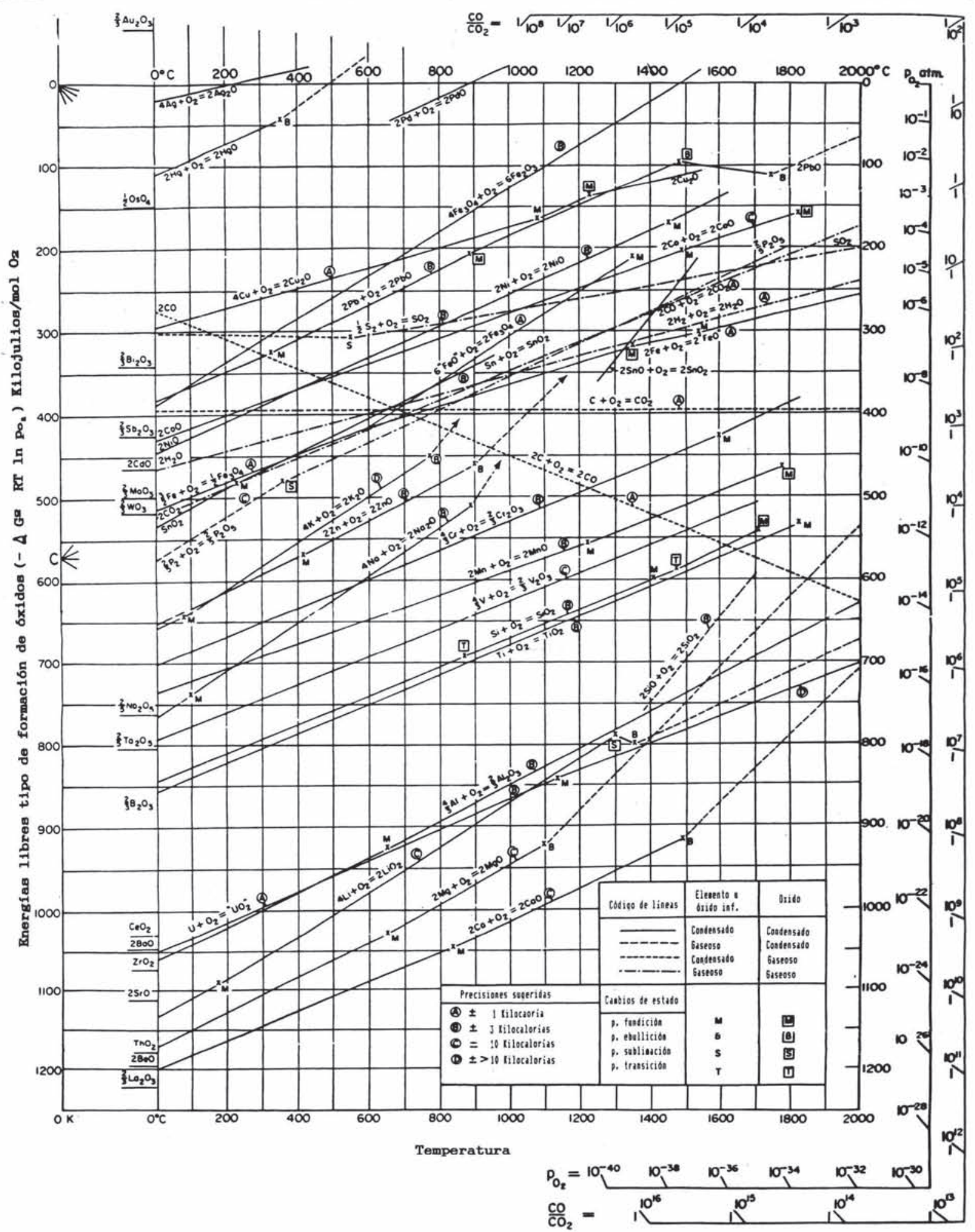

Fig. 1. Diagrama de Ellingham para la formación de óxidos según Gilchrist (1989). Los estados tipo son las fases puras condensadas y los gases a una atmósfera de presión. Las escalas para los valores de $\mathrm{p}_{\mathrm{O} 2}$ y $\mathrm{CO} / \mathrm{CO}_{2}$ están indicadas en el margen derecho y radiando hacia los focos marcados en el eje de Temperaturas. Allí donde no se conocen los valores con exactitud, o donde su inclusión pudiera dar lugar a confusión, se indica el óxido con su fórmula en el valor aproximado de $\mathrm{G}^{\mathrm{O}}$ a $0^{\circ} \mathrm{C}$.

T. P., $51, \mathrm{n}^{\circ} 1,1994$ 
partir de esa temperatura lo es el C. Este hecho puede ser de gran utilidad para entender el funcionamiento de los hornos prehistóricos, aunque no debe caerse en el error de pensar que las cosas son tan sencillas: todo lo que estamos diciendo está basado en la termodinámica, y no tiene en cuenta otro gran número de factores como la cinética o la porosidad de los sólidos.

Con todo, podemos usar como referencia un modelo para la reducción moderna como el siguiente (Faust, 1986: 78):

1. El aire inducido, con una temperatura en torno a los $1000^{\circ} \mathrm{C}$, reacciona con el carbón para producir monóxido de carbono. La temperatura de la llama ronda los $2200^{\circ} \mathrm{C}$. La formación del monóxido de carbono a partir del dióxido de carbono se completa aproximadamente un metro por encima de las toberas.

$$
\mathrm{C}+\mathrm{O}_{2} \rightarrow \mathrm{CO}_{2} \stackrel{\mathrm{C}}{\rightarrow} 2 \mathrm{CO}
$$

2. La reducción empieza en lo alto del horno, a una temperatura en torno a los $450^{\circ} \mathrm{C}$. Primero, la hematita se reduce a magnetita, y a partir de aquí a wüstita. La reducción se completa a temperaturas por debajo de los $900^{\circ} \mathrm{C}$, lográndose reducir tan sólo el $65 \%$ del FeO (así se explica la riqueza de hierro de las escorias antiguas).

$$
\begin{aligned}
& 3 \mathrm{Fe}_{2} \mathrm{O}_{3}+\mathrm{CO} \rightarrow 2 \mathrm{Fe}_{3} \mathrm{O}_{4}+\mathrm{CO}_{2} \\
& \mathrm{Fe}_{3} \mathrm{O}_{4}+\mathrm{CO} \rightarrow 3 \mathrm{FeO}+\mathrm{CO}_{2} \\
& \mathrm{FeO}+\mathrm{CO} \rightarrow \mathrm{Fe}+\mathrm{CO}_{2}
\end{aligned}
$$

3. $\mathrm{El}$ resto de $\mathrm{FeO}$ se reduce por encima de los $1000^{\circ} \mathrm{C}$ (recuérdese que en la actualidad los altos hornos superan el punto de fusión del hierro, a $1.500^{\circ} \mathrm{C}$ ) de acuerdo con la ecuación:

$$
\mathrm{FeO}+\mathrm{C} \rightarrow \mathrm{Fe}+\mathrm{CO}
$$

$\mathrm{Al}$ mismo tiempo, también es interesante recordar que, tal y como se deduce del examen de algunos hornos, a veces el proceso fue demasiado lejos, produciéndose cantidades considerables de carburo de hierro (Tylecote, 1.987: 152).

$$
3 \mathrm{Fe}+\mathrm{C} \rightarrow \mathrm{Fe}_{3} \mathrm{C}
$$

\section{LA REDUCCIÓN EXPERIMENTAL: ENCUADRE HISTÓRICO}

Las fuentes fundamentales para el estudio de la metalurgia del hierro son las escorias (de re- ducción y de forja) y las piezas terminadas (McDonnell, 1989: 373) pero ésto no debe hacernos olvidar la información que viene de los restos de hornos y de la arqueología experimental.

Esta última ha girado fundamentalmente en torno al cobre y hierro, mientras que se ha trabajado muy poco en otros campos como los otros metales o, aún menos, los minerales sulfurosos.

Los primeros trabajos se centraron fundamentalmente en el estudio del cobre. Deben destacarse los de Cusishing, a finales del s. XIX, y los de Gowland, a principios del s. XX.

$\mathrm{El}$ examen de los restos romanos de trabajo de hierro en los años treinta motivó reconstrucciones que permitieran comprender ese material, estando entre las primeras las de Gilles en Alemania en los años cincuenta, seguido de Wynne y Tylecote en Inglaterra, al tiempo que en el campo de la Antropología se trataba de registrar técnicas de trabajo casi perdidas en Africa y Asia antes de que cayesen en el olvido.

De gran interés han sido las experiencias realizadas en Polonia, en Nova Slupia (Nosek, 1985: 166), y en Dinamarca, donde se ha llegado a crear una tradición cultural valiosa por sí misma. Ambos centros han dejado bien claro que es necesario una gran habilidad y que la clave del éxito está en la manipulación del contenido.

La realización de éstos y otros trabajos sobre el hierro desde la II Guerra Mundial ha hecho aumentar considerablemente nuestra comprensión del problema, hasta el punto de que Tylecote y Merkel (1985: 13) llegaron a afirmar que para muchos es hoy más familiar la reducción del hierro que la del cobre.

Con todo, autores como Budd y otros (1992: 1), hablando de la reducción en los comienzos de la metalurgia, se quejan todavía del excesivo hincapié en los análisis puramente composicionales, lo que, por un lado, deja relativamente poco comprendidas las técnicas prehistóricas de manipulacion y extracción del metal y, por otro, da lugar a unos modelos que empiezan a resultar incompatibles con algunos de los trabajos que están volviendo a centrarse recientemente en la metalurgia extractiva y la producción de piezas.

\section{TIPOS DE REDUCCIÓN Y TIPOLOGÍA DE HORNOS}

Rostoker y Dvorak (1990: 153) reconocen tres procesos a lo largo de la historia para la 
consecución de hierro bruto. El primero, la fragua catalana, consiste en la reducción directa en fase sólida del mineral lográndose un metal con un alto contenido en carbón. Este sistema pervivió en algunas regiones hasta el s. XX (aunque estaba ya en desuso en Europa y Norte América a mediados - finales del s. XIX). Con el segundo tipo, con horno de afino, se lograba una colada de hierro fundido prácticamente descarburizado, por lo que sólo se desarrolló una vez que el paso de mineral de hierro a hierro colado fue comercial (se considera que el inicio de este tipo de producción a escala comercial en Europa es rastreable ya a inicios del s. XVI). La tercera técnica fue el pudelado, desarrollada a finales del s. XVIII en Inglaterra y que desplazó a los otros dos sistemas. Suponía un abaratamiento de costes en la obtención de un metal de primera fundición similar al obtenido por el segundo método.

Las condiciones del horno en que se efectúe la reducción (durante la Prehistoria, por supuesto del primer tipo descrito en el párrafo anterior) han de ser muy reductoras para evitar la reoxidación del hierro ya reducido. Tanto es así, que es en ello en lo que se basa Tylecote (1986: 124$)$ para explicar la aparición tardía del hierro en la historia de la metalurgia. También según este autor (Tylecote, 1987: 151) esta exigencia, junto con el hecho de que el metal no llega a fundirse, no sólo da lugar a dificultades de trabajo sino tal vez incluso a una nueva filosofía en la que la forja era un requisito imprescindible para consolidar el hierro reducido y eliminar la escoria residual (frente a lo que pasaría a finales de la Edad del Bronce, momento en que poca forja habría sido necesaria), aunque ésto es algo todavía por evaluar propiamente.

En 1956, H. H. Coghlan publicó un intento de clasificación de estos hornos en base simplemente a un criterio morfológico (Coghlan, 1956: 86). Él reconocía tres tipos: un horno formado por un simple hoyo en el suelo, un horno cupuliforme y el vertical. También pensaba que habría que incluir la diferenciación entre ventilación inducida o forzada con fuelles. De todas formas, él mismo reconocía que los últimos hallazgos suponían una cantidad confusa de tipos.

R. F. Cleere (1972) revisó esta clasificación, que ya daba bastantes problemas, entre ellos el de considerar la posibilidad de un hoyo abierto como horno, cuando los experimentos realizados por Tylecote en los años cincuenta demostraron ya que tan sólo cuando el hoyo estaba cubierto se podía llevar a cabo la reducción (Cleere, 1972: 10).

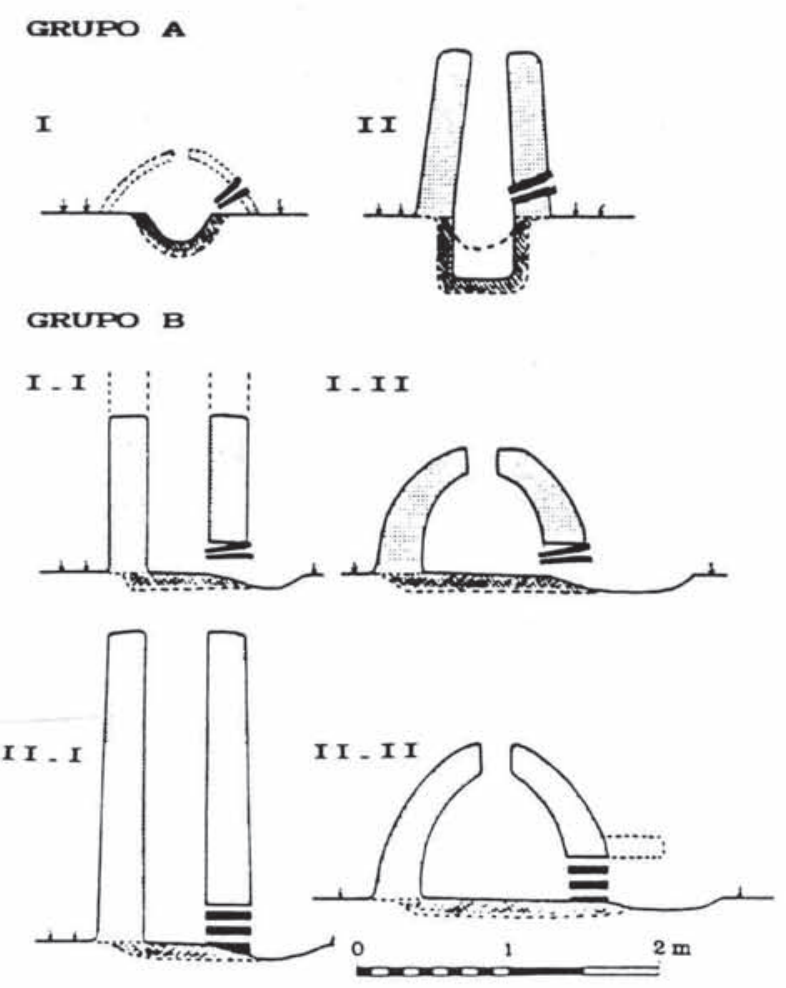

Fig. 2. Clasificación de los hornos para la reducción de hierro según Cleere.

Si seguimos a Cleere (1972: 21 a 23), se puede encuadrar una descripción de un horno especificando forma, emplazamiento del fuego y la manera de eliminar la escoria (ésta es probablemente su aportación principal). La clasificación de Cleere (Fig. 2) consta de un complejo cuadro de grupos y subgrupos que tampoco llega a cuadrar totalmente (tal vez por su excesiva atención al aspecto formal de los hornos). Distingue primero entre hornos sin y con sistema de extracción de escoria. Los primeros caracterizados por no extraerse la escoria del horno durante el proceso, tener el hogar por debajo del suelo y funcionar con ventilación forzada; y los segundos por tener sistema de extracción de escoria, el hogar al nivel del suelo y constar de una superestructura. Después, subdivide entre hornos con superestructura cilíndrica 
o sin ella, para el primer caso, y cuatro subtipos en el segundo según tuvieran una superestructura cilíndrica o hemiesférica y según tuvieran o no ventilación por fuelles.

En principio, el tipo de los Montes de Santa Cruz sería el A-II de Cleere, mientras el caso de Moesgaard no acaba de encajar ya que no tiene salida de escoria al exterior, pero su zona de trabajo queda por encima de la superficie del suelo (y no por debajo, lo que constituye una de las características de los tipos A).

Tylecote (1987: 151) simplifica la clasificación, se limita a hablar de hornos de cuenco y vertical, y acepta tan sólo una diferenciación de tipos por tener o no salida de escoria al exterior. Es una clasificación más genérica que la de Cleere pero que puede resultar más útil al dejar más sitio para consideraciones tecnológicas (que él no llega a incorporar, por otro lado). De hecho, sus estudios permiten encuadrar mejor el tipo de horno con que se experimentó en Moesgaard.

En cualquier caso, no se puede esperar una única explicación válida para todos los casos, a pesar de que cuanto se diga debe poder corresponderse con la referencia única del comportamiento teórico de los materiales dado por las Ciencias Naturales. No estamos sólo ante una difusión de tipos al estilo tradicional de la arqueología, sino que además tenemos una adecuación de técnicas aprendidas con distintas variantes locales (cuyos motivos pueden ser muy complejos).

En su búsqueda de paralelos, Tylecote y Merkel (1985: 9) llegan a hablar de un "paralelismo interesante" entre hornos nórdicos y un tipo de horno de cuenco de los burundi, en Africa Oriental. Pero no deja de quedar la duda de si no se tratará de un caso similar al que se podría hacer entre los hornos nórdicos y los verticales de los hoya, al NO de Tanzania (Schmidt, 1983: 421), también con un parecido morfológico grande pero con sistemas de funcionamiento muy diferentes como pone de manifiesto el que el hierro se mantiene en estado sólido en el caso danés, mientras que se defiende la licuación en el caso africano (Schmidt, 1983: 425).

En definitiva, se trata de cuestiones de tecnología que dejan unas huellas muchas veces difíciles de interpretar, y que resultan cuando menos complejas de tratar desde un punto de vista tipológico tradicional, sin contar con su técnica de trabajo.

\section{EL HORNO VERTICAL CON HOYO DE ESCORIA}

En general, estos hornos se caracterizan por tener un escorial enterrado, en vez de una salida externa de escoria, sobre el que se encuentra una "chimenea" de arcilla refractaria que constituye el horno en sí. La zona principal de combustión queda en la base de esta chimenea, por encima o ligéramente por debajo del nivel del suelo según el tipo concreto de horno de que se trate.

La reconstrucción con que se trabajó en Moesgaard (Fig. 3) consistía en un hoyo invasado de planta circular excavado en el suelo, cerrado por un anillo modelado en arcilla refractaria cuya función era permitir que la escoria fundida bajara al escorial al tiempo que mantenía el hierro ya reducido en la zona superior, facilitando así la separación de ambos (este estrangulamiento del espacio disponible no se ha encontrado en la documentación manejada sobre los otros tipos mencionados). Por encima se construyó una chimenea troncocónica de ca. 1'10 m. de altura con cuatro perforaciones en la base de ca. $4 \mathrm{~cm}$. de diámetro situadas en los extremos de los ejes ortogonales de la circunferencia de base.
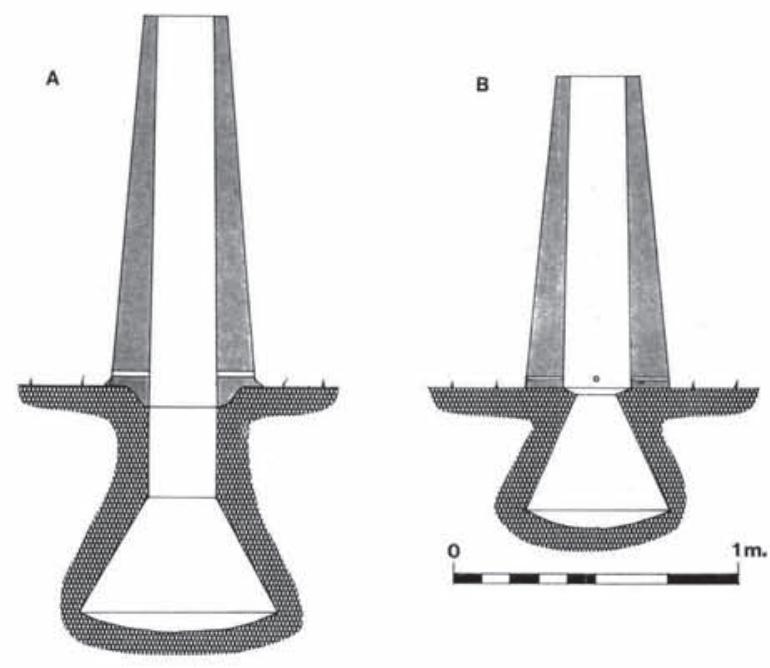

Fig. 3. Reconstrucción del horno vertical con hoyo de escoria a partir de los hallazgos realizados en Dinamarca A) según Thomsen y B) tal y como se reconstruyó para la experiencia descrita.

En los hornos de este tipo, se deja que combustible y mineral, generalmente dispuestos en 
estratos, vayan bajando juntos por la chimenea del horno (como punto de comparación, puede recordarse que el tipo de horno catalán trabaja con el mineral en un sitio y el combustible en otro, sin darse ningún tipo de alternancia).

Según Tylecote (1986: 130), una de las ventajas del horno vertical es que puede funcionar con corriente natural de tener la altura suficiente. El principio es bien simple: el aire asciende por la diferencia de densidad que se produce entre el aire frío y el caliente; cuanto mayor sea la diferencia de temperatura y más alta sea la columna, mayor será el flujo que se consiga. Haynes Cleere (1970) encontró que se puede alcanzar una temperatura de hasta $1150^{\circ} \mathrm{C}$ en el fondo de un horno de sección cuadrada de $22 \mathrm{~cm}$. de lado y sólo $25 \mathrm{~cm}$. de altura. Ahora bien, el carbón y el mineral entorpecen la corriente, y hay que aumentar la altura para mantener el mismo flujo. Tylecote da también como prueba de la bondad de ésto el que la mayoría de los hornos usados por los primitivos actuales tienen una altura de 2-3 m.

Por lo general, sólo se documenta arqueológicamente la parte enterrada, el hoyo, siempre relleno por un cilindro de escoria (la llamada schlackenklotz).

Tal vez el yacimiento más famoso con documentación de este tipo sea el de los Montes de Santa Cruz, al SO de Varsovia (Nosek, 1985). Se trata de una enorme región de reducción de hierro (sometida a investigación sistemática desde 1955) que ha aportado cerca de 3.000 yacimientos relacionados con la reducción del hierro, cada uno con una media de unos 100 hornos (el yacimiento más grande tiene hasta 123). Se han excavado unos 120 de ellos, fechándose en los primeros cuatro siglos de nuestra Era.

Las excavaciones han permitido determinar dos tipos de taller (Nosek, 1985: 165 a 166): en unos casos se trata de hornos pequeños distribuídos aleatoriamente entre los lugares de habitación. En otros se trata de grandes zonas preparadas para la reducción: aquí, los yacimientos consisten en escoriales dispuestos paralelamente en filas generalmente con un espacio vacío en medio formando una especie de pasillo, y una media de tres hornos a cada lado de dicha zona libre. El origen de estos escoriales está en la destrucción del horno para sacar el hierro bruto una vez terminada la reducción, dejándose la escoria in situ; para un nuevo proceso de reducción, se construía otro horno al lado de donde estuvo el anterior. Los hornos venían a tener una altura de unos 100-110 cm., aunque también hay otros de $80-100 \mathrm{~cm}$.

Otro yacimiento que ha proporcionado hornos muy similares a los polacos es Blansko, en Checoslovaquia (Souchopová, 1989: 150). Sin embargo, este caso (que responde bastante bien al tipo A2 de Cleere) también pueden encontrarse diferencias, como las "abrazaderas de tobera" que, como se ve en Velkomoravske, funcionaban como una boca cónica que facilitaba la inserción de la tobera.

El caso danés es muy similar a los dos ya mencionados, como se puede deducir de las descripciones que se han ido dando. Las reconstrucciones que se hacen hoy en Dinamarca están basadas en la que hizo Thomsen en 1963 (Fig. 3), modificada fundamentalmente reduciendo la profundidad y la forma del escorial.

Los hallazgos de hornos de este tipo se sitúan fundamentalmente en la zona Occidental de Jutlandia, claramente relacionados con la mayor concentración de limonita o hierro de pantano. Un ejemplo de la excavación de estos hornos es el que da Liv Appel (1986) hablando del yacimiento de Maglegaard. Su cronología los sitúa en los cuatro primeros siglos de nuestra era, al igual que otro tipo de horno, más pequeño, con planta de herradura y chimenea baja, y del que no hablaremos ya aquí, que se vincula con la explotación de menas de reducido tamaño esparcidas por otras zonas del suelo danés.

La experiencia realizada en Moesgaard se vincula, precisamente, al estudio e intento de comprensión de esos hallazgos de hornos verticales con hoyo de escoria.

\section{EL CONTEXTO CULTURAL}

Antes de describir la reducción experimental en la que se tuvo la oportunidad de participar, puede ser conveniente recordar algunos aspectos de la Edad del Hierro en Dinamarca, dado que el concepto que hay detrás de ella es muy diferente cronológica y culturalmente al que encierra para la Prehistoria Española. Una referencia más amplia puede encontrarse en Joorgen Jensen (1982) o en Lone Hvass (1980); para aspectos puntuales puede usarse el diccionario arqueológico de Hedeager y Kristiansen (1985). 
En sentido amplio, la Edad del Hierro (Jernalder) se fecha entre el 500 a.C. y el 1050 d.C. (Cristianización y unificación de Dinamarca), y suele dividirse de dos formas. De acuerdo con una de ellas, se habla de cuatro períodos: el Preromano (500 a.C. hasta el cambio de Era), el Romano (hasta el 400 d.C.), el Germánico (hasta el 800 d.C.) y el Viquingo (800-1050 d.C.). Y de acuerdo con la otra, se agrupan esos cuatro períodos de dos en dos dando lugar a una Alta y Baja Edad del Hierro. Nos centraremos en el primero de los períodos de esta segunda clasificación.

En torno al 500 a.C. parece detectarse una crisis que deja paso, en los siglos siguientes, a una sociedad agraria con un potencial considerable de expansión, identificándose para el cambio de Era en toda Dinamarca síntomas claros de crecimiento económico que culmina en los ss. III y IV con la aparición de aldeas formadas por granjas con una economía diversificada que proporcionaba buenas posibilidades para las actividades secundarias.

Uno de los cambios más importantes tuvo lugar en la estructura de las aldeas, de carácter móvil dentro de una área de explotación bien definida, lo que permitía una mejora de la tierra con la basura humana de la ocupación anterior (como puede verse en Groontoft, NO de Jutlandia). El comercio también fue importante (Dinamarca estaba vinculada a una amplia red de intercambio desde el II Milenio a.C.), con competitividad entre centros locales, cambios de rutas comerciales y de focos de comercio paralelos a cambios de centros políticos y a numerosos intentos de unificación (este proceso dará lugar, ya en el Período Viquingo, a la aparición de los primeros centros urbanos con el consiguiente desarrollo de la actividad artesanal). Otro aspecto destacable son las ofrendas, tanto de objetos (baste recordar el famoso caldero de Gundestrup) como sacrificios humanos.

En cuanto al hierro, las primeras huellas de minería se remontan a los ss. V-IV a.C. (p.e. Bruneborg). Se trata de explotaciones de hierro de pantano, muy abundante en el Sur y Oeste de Jutlandia, introduciéndose un cambio fundamental en la metalurgia danesa, hasta entonces absolutamente falta de materia prima.

Durante los primeros cinco siglos de la Edad del Hierro, la extracción debió ser modesta, teniéndose que complementar con importaciones. $\mathrm{Al}$ principio, se realizan sólo pequeños objetos como agujas; las fíbulas de hierro empiezan a aparecer hacia el 200-100 a.C., y no es hasta el cambio de Era que los objetos grandes empiezan a hacerse frecuentes. El desarrollo de la producción de hierro tuvo lugar a partir del s. II d.C. en relación con mejoras técnicas como el horno vertical, que se seguirá usando hasta mediados del I Milenio. Estos hornos suelen aparecer en grupos, como en Drengsted, donde se llegó a encontrar hasta doscientos de ellos.

\section{LA REDUCCIÓN EXPERIMENTAL DE HIERRO}

La experiencia de Moesgaard es similar a otras realizadas sobre hornos del mismo tipo en países como Checoslovaquia (Souchopová, 1989) o Polonia (Nosek, 1985), y, al igual que en estos casos, cubre no sólo un aspecto científico, sino también divulgativo y de entretenimiento (en Nosek, 1.985: 169 llega a tocar incluso lo publicitario). El trabajo consistió en reducir mineral de hierro en dos hornos verticales forzando el soplo en uno con fuelles dobles y en otro con una bomba de aire.

Se empleó limonita como punto de partida (éste es el mineral empleado en la Prehistoria Danesa). Esta se obtuvo en las tierras del Oeste de Jutlandia y se llevó a Moesgaard en bloques, tal y como los sacó la pala, y aún con restos de vegetación (recuérdese que se trata de mineralizaciones de superficie). En los experimentos polacos (Nosek, 1985: 166) el mineral empleado fue hematita mezclada con dolomita, siderita y pyrita, mientras que en el caso checo (Shouchopová, 1989: 150) era limonita y hematita (encontradas en los alrededores de los yacimientos) mezcladas con mineral de alto contenido en hierro, proporcionado por una empresa moderna, para evitar un fracaso de la experiencia por pobreza de mena.

La primera labor fue proceder al tostado o calcinación del mineral. Para ello se dispuso una plataforma de troncos en un espacio llano y abierto sobre la que se colocó el mineral cubierto con más leña, y se le prendió fuego. Es decir, se procedió a un mero calentamiento del mineral en atmósfera oxidante.

Según Tylecote (1987: 53), es indiferente hacer el tostado sobre plataformas de leña al aire libre o bien en pequeñas hondonadas. Después de la Edad Media, se usaron ya hornos más per- 
manentes para el tostado (ver, por ejemplo, Linsley y Hetherington, 1978), pero lo realmente importante es facilitar al máximo la corriente de aire (si bien no hace falta usar fuelles, ya que el rango de temperaturas necesario es de tan sólo $500-800^{\circ} \mathrm{C}$ ).

Es difícil creer que en la Antigüedad se supiera lo que realmente pasaba durante la calcinación, pero sus beneficios son muy claros, y probablemente pueda considerarse como usual en la tecnología metalúrgica antigua. Ya en el caso del cobre algunos minerales, como los sulfuros primarios con arsénico no pueden ser reducidos directamente sino que es necesario una calcinación que convierta los sulfuros en óxidos metálicos (Budd et alii, 1992: 4). Por otro lado, los carbonatos también mejoran y se consigue el secado del mineral así como aumentar su porosidad (con lo que se permite una mejor penetración de los gases reductores).

Los bloques de mineral que se obtuvieron al final presentaban un aspecto poroso, seco y fácilmente deleznable. Se procedió a desmenuzarlos con un martillo con el fin de conseguir fragmentos inferiores al tamaño de medio puño para facilitar la reducción, pero sin llegar a moler totalmente el mineral, ya que probablemente verterlo en polvo en vez de en bloques hubiera supuesto cegar el tiro del horno.

El otro elemento importante a preparar es el carbón. En Moesgaard se buscó que éste fuera "pesado" (se usó madera de tilo). En Polonia (Nosek, 1985: 167 y 168) se analizó el carbón que quedaba por debajo de las escorias y se determinó que se trataba de una mezcla de carbón obtenido de maderas duras como haya y roble y de coníferas como pino, abeto y alerce. También se encontró carbón de álamo y tilo. Posteriormente, la reducción experimental descrita por Nosek demostró que la proporción ideal de haya a pino era de 1:2, y que la de mineral a carbón era igualmente de 1:2. Esto supone que era necesario contar con una gran cantidad de madera para poder disponer del carbón suficiente: seis veces el peso del carbón vegetal en madera (Waldbaum, 1989: 118).

Antes de iniciar la reducción, se hace necesario encender y precalentar el horno. Simplemente se llenó el horno de paja que se prendió por arriba y por las cuatro toberas, alimentándose el fuego con algo de carbón durante cuatro horas (Nosek (1985: 167) comenta que ellos necesitaron doce). Con ello se consiguió secar y endurecer el horno así como ir aumentando la temperatura en su interior, antes de empezar a cargar realmente el horno.

Una vez bien seca la estructura, empezó el trabajo realmente relacionado con la reducción. Primeramente, se siguió quemando carbón hasta alcanzar temperaturas en torno a los $900^{\circ}$ $\mathrm{C}$ en la base de la chimenea, y se dejó que el carbón bajase hasta dejar unos dos tercios del horno libres. En ese momento se llenó completamente de mineral. Cuando éste ya había bajado algo, se prendió fuego a los gases que salían por la boca de la chimenea (esta llama quedó ya encendida durante todo el proceso), y una vez quedaron de nuevo dos tercios de espacio libre, se volvió a cargar completamente con carbón pero, a diferencia de la primera vez, se empleó aire forzado durante veinte - treinta minutos con el fin de aumentar la temperatura del horno hasta unos $1200^{\circ}-1300^{\circ} \mathrm{C}$ (acelerándose también el ritmo de combustión del carbón) hasta volver a tener los dos tercios del espacio libres, tras lo que se paró el soplo forzado y se volvió a cargar el horno con carbón. Se trabajó, por tanto, con una combinación de alimentación inducida y forzada de aire. Nosek (1985: fig. 4) da una representación gráfica de las temperaturas medias dentro de las distintas zonas de un horno similar al descrito.

A partir de este momento, el ritmo de trabajo se estructuró en tres etapas. Primeramente, se cargaba el horno de carbón que se dejaba consumir; después, se cargaba de mineral (ca. $10 \mathrm{Kg}$.) y, una vez había bajado dejando dos tercios de la chimenea libres, se volvía a cargar de carbón (ca. $8 \mathrm{Kg}$.) y se forzaba el tiro hasta que aquél se consumía. Los períodos de tiro forzado duraban unos veinte - treinta minutos, mientras que los períodos sin soplo podían durar entre tres y seis horas. La intención era repetir ininterrumpidamente esta secuencia hasta que la escoria rebosase y cegara las piteras de alimentación de aire, pero en la práctica, el horno tuvo que ser apagado después de tres días de trabajo agotador. Se habían empleado al final ca. 200 $\mathrm{Kg}$. de mineral y $750 \mathrm{Kg}$. de carbón por horno.

Hay varias razones para hacer todo ésto de esa forma. El hierro reduce a temperaturas ligeramente inferiores a los $1000^{\circ} \mathrm{C}$, difíciles de alcanzar en una estructura que no esté seca, por lo que es inútil intentar obtenerlas con cargas grandes de carbón hasta que no se ha cocido la arcilla del horno e, igualmente, no sirve de nada 
verter mineral hasta que no se ha llegado a calentar toda la estructura a la temperatura de trabajo.

Por otro lado, es fundamental contar con una corriente de aire cargado de carbono, ya que es éste el agente reductor. Esta era la utilidad de la llamarada: al crearse una fuente de calor en la zona superior se lograba absorber aire por la base a través de las piteras. Esto bastaba para mantener las condiciones reductoras, pero para eliminar la escoria era necesario emplear fuelles con el fin de alcanzar temperaturas en torno a los $1200^{\circ} \mathrm{C}$.

Antes de usar los fuelles, el mineral se ha ido reduciendo dando lugar a hierro metálico (que origina una lupia o esponja de hierro dulce por crecimiento de cristales en sólido de acuerdo con mecanismos de difusión atómica) y escoria sólida muy mezclada con éste. Para separar lupia y escoria se fuerza el tiro aumentándo la temperatura lo suficiente para fundir ésta, con lo que baja al escorial por gravedad, mientras que el hierro, al no fundirse más que de manera excepcional, queda retenido por el estrangulamiento que sirve de boca al escorial.

El sistema de soplo forzado consistió para uno de los hornos en cuatro fuelles dobles dispuestos ortogonalmente alrededor del horno, con los boquereles horizontales y embocados hacia piteras de unos cinco centímetros de diámetro. La conexión entre los boquereles y las piteras no era directa, sino que estaban separados unos veinte centímetros. Este sistema de fuelles dobles permite conseguir sin excesivo esfuerzo un soplo fuerte y continuo de aire, lo que es bastante importante, ya que al estar las piteras enfrentadas dos a dos, la suspensión de la entrada de aire por una de ellas implicaba que el flujo proveniente de la opuesta saldría al exterior por ella, con los consiguientes riesgo y pérdida de calor. En el otro horno, el sistema fue similar salvo que los cuatro fuelles dobles se sustituyeron por una bomba eléctrica de aire (resulta curioso reseñar que ésta proporcionaba una corriente menos intensa que los fuelles, lo que se tradujo en la consecución de temperaturas algo inferiores).

No hay muchas pruebas del empleo de estos fuelles, pero sí suficientes. Ténganse en cuenta por ejemplo, aparte de la necesidad técnica, los hallazgos checos de boquereles para hornos similares al de Moesgaard descritos por Souchopová (1989: 160). De todas formas, quedan aún cuestiones en el aire, como la de cuántos boquereles se emplearon en el caso checo (una cuestión importante para determinar el tipo de técnica de producción empleada). Por otro lado, en Polonia se hacía entrar el aire inducido con una inclinación de $30^{\circ}$ en relación a la generatriz del horno (de manera similar al caso checo), y se observó que la realización de un pequeño agujero de unos $2 \mathrm{~cm}$. de diámetro en frente de la entrada del aire, justo por encima del nivel de la tobera, permitía la salida de algunos gases cuando se soplaba y se traducía en un aumento de la zona reductora (Nosek, 1985: 168 y fig. 5). En Moesgarde, como se ha dicho, los fuelles estaban colocados horizontalmente y separados del horno, además de dispuestos dos a dos y soplando todos al mismo tiempo precisamente para evitar la salida de aire, lográndose reducir hierro igual que en el caso polaco. Por otro lado, en una experiencia similar realizada en la Escuela de Ingeniería Técnica de Copenague (D.T.H.) se hicieron cuatro piteras pero sólo se usaron dos fuelles no enfrentados, con lo que hubo salida de gases pero no hubo reducción (con todo, en la D.T.H. se cometieron muchos errores y no puede saberse qué incidencia tuvo este detalle).

El aire se insuflaba frío, sin precalentamiento previo como en el caso (que no deja de ser controvertido) de los hornos africanos de los Haya (Schmidt, 1983: 425).

Una vez se dio por finalizado el proceso de reducción, se dejó enfriar el horno durante una semana, y se procedió a excavarlo a la manera tradicional.

El resultado fundamental es que se consiguió reducir hierro, con lo que se cumplió uno de los objetivos fundamentales. Otra cuestión es la calidad de este hierro, de lo que hablaremos más adelante.

\section{ESTUDIO METALOGRÁFICO}

Terminada la experiencia, se realizó en España un estudio metalográfico de las muestras buscando fundamentalmente comprobar el resultado de la reducción y poder disponer de muestras de referencia que aplicar al estudio de las piezas históricas, ya que es muy difícil encontrar en los manuales metalográficos modernos ejemplos de metales con el tipo de defectos 
que pueden surgir de un trabajo como el llevado a cabo.

Se tomaron cuatro muestras pensando en las cuatro fases fundamentales del proceso de reducción: el mineral, la reducción del hierro (FrI), la forja para la eliminación de restos de escoria (FrII), y la forja para la realización de piezas concretas (FrIII). Por ello, se montaron para la realización de metalografías muestras de mineral, de la masa de hierro dulce, de hierro semimartilleado, y de una barra de hierro hecha en forja.

El mineral. Poco es lo que se puede decir de la muestra mineral dentro de este artículo. Por lo que ahora nos interesa, prácticamente sólo nos permite ilustrar esa fase del proceso. Se trata de una limonita (junto con carbonatos y hematites, uno de los tipos fundamentales de mineral de hierro). Limonita es una palabra general para los óxidos de hierro con agua $(\mathrm{FeO} . \mathrm{OH})$; son minerales indeterminados, precipitados de aguas ricas en hierro de pantanos y lagos, con contenidos varios de goetita, lepidocrocita, materia amorfa y otros minerales (como granos de cuarzo).

Abundan principalmente en las zonas húmedas de Europa, pero también se han identificado en España e Israel. Es importante precisar aquí que, aunque en la bibliografía arqueológica se suele hablar de depósitos bien conocidos como Weald en Inglaterra o los Montes de Santa Cruz en Polonia, hay muchos más depósitos, muchas veces demasiado pobres para el uso moderno pero que pudieron ser usados en la Antigüedad. Tylecote (1987: 53) llega a sugerir que, en ausencia de mejor cosa, puede que la gente primitiva se planteara usar minerales con un contenido en Fe de sólo un 1\%.

El propio mineral es el origen principal de impurezas tales como $\mathrm{Ca}, \mathrm{Al}, \mathrm{Mg}, \mathrm{Mn} \mathrm{P}$, hierro mineral que pueden aparecer en el hierro reducido. Este sale del horno con una estructura porosa, con los poros llenos de escoria, pero la escoria no aparece en el mineral, sino que es necesario que haya habido una reducción para poder encontrarla.

Una de las impurezas principales que aporta el mineral (Tylecote, 1987: 52) además de azufre y arsénico, es el fósforo. Aparece en los metales reducidos en sólido, y puede endurecer y volver quebradizo el metal. Hoy se consigue que pase a la escoria, pero en el proceso de reducción só- lida aproximadamente un cuarto de la cantidad original en el mineral pasa a la masa metálica; teniendo en cuenta que el mineral puede llegar a tener hasta un $2 \%$ de $\mathrm{P}$, ésto significa mucho $\mathrm{P}$ en el metal (Tylecote, 1987: 52). El Manganeso no se reduce y pasa a la escoria, pero puede aparecer hasta un $2 \%$ de As en el metal. $\mathrm{Cu}, \mathrm{Ni}, \mathrm{Ag}$ y Au también pueden pasar al metal.

Por otro lado, los minerales de Hierro pueden ser óxidos o sulfatos, derivando muchos óxidos de los sulfuros. Así, el azufre es un elemento residual en el hierro que puede llegar hasta el 1\%. Según Tylecote (1987: 48) ésto puede ser sobrevalorado y considera que su presencia es más negativa en el hierro moderno (que ha pasado por una fase líquida, concentrándose en los límites de grano y aumentando la fragilidad del metal) que en el hierro prehistórico, obtenido por reducción en sólido, y que puede ser forjado aún con contenidos de $\mathrm{S}$ de hasta un $1 ' 0 \%$ (aunque con cuidado).

Muestra FrI (Lám. II: a y b) la fase de hierro puede suponer el $80 \%$ y la escoria el $20 \%$. La fase de hierro es muy heterogénea, cambiante de $\mathrm{mm}$ a $\mathrm{mm}$, con ferrita y perlita (la perlita está formada por lamellae de cementita, $\mathrm{Fe}_{3} \mathrm{C}$, en hierro $\alpha$, llamado ferrita), generalmente formando estructuras de Widmanstätten. El contenido en carbono está probablemente entre el 0.2 a 0.4 (la ferrita contiene un $0 \% \mathrm{C}$, la perlita 0.8 ). La escoria está formada por dendritas de óxido de hierro $(\mathrm{FeO})$ en una matriz obscura vidriosa conteniendo todas la mayor parte de las impurezas del mineral tales como calcio, potasio, aluminio, silício, manganeso, fósforo. La es-

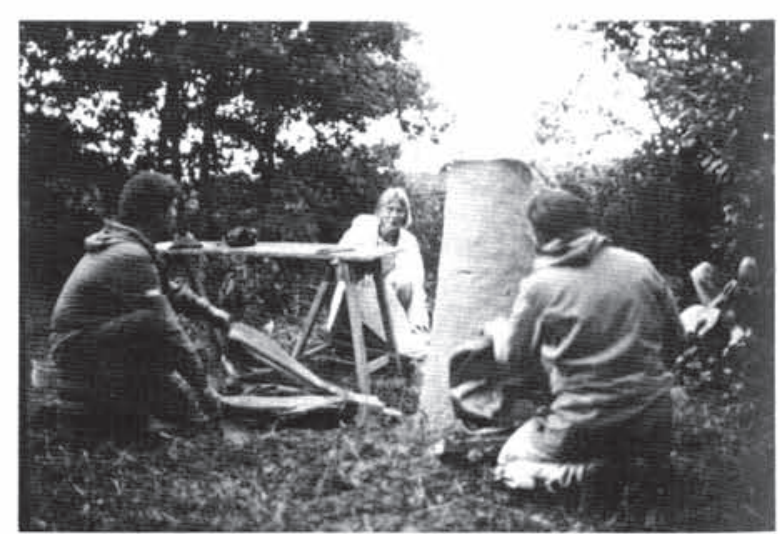

Lám. I. El horno vertical durante el proceso de reducción. 
A

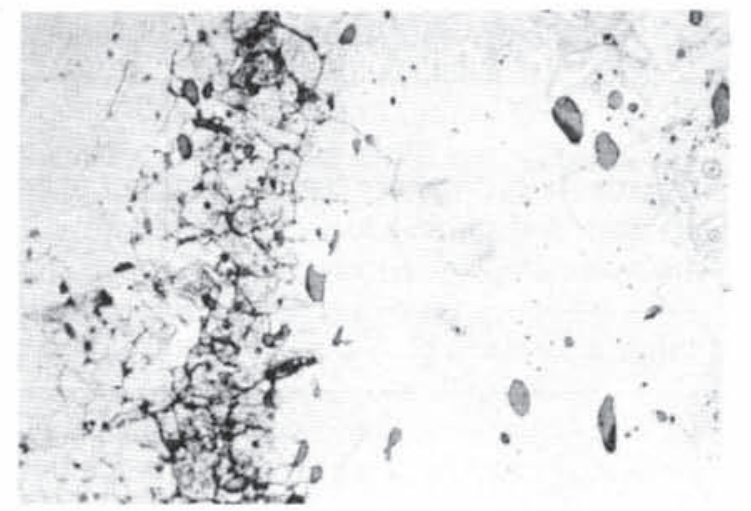

C

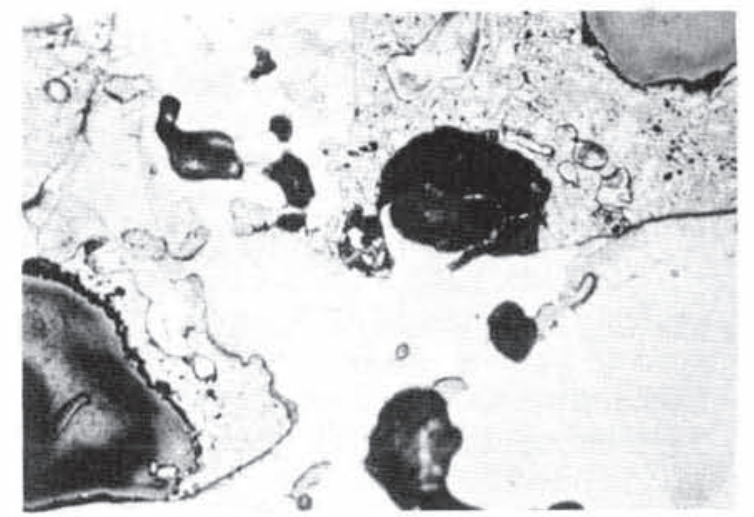

$\mathrm{E}$

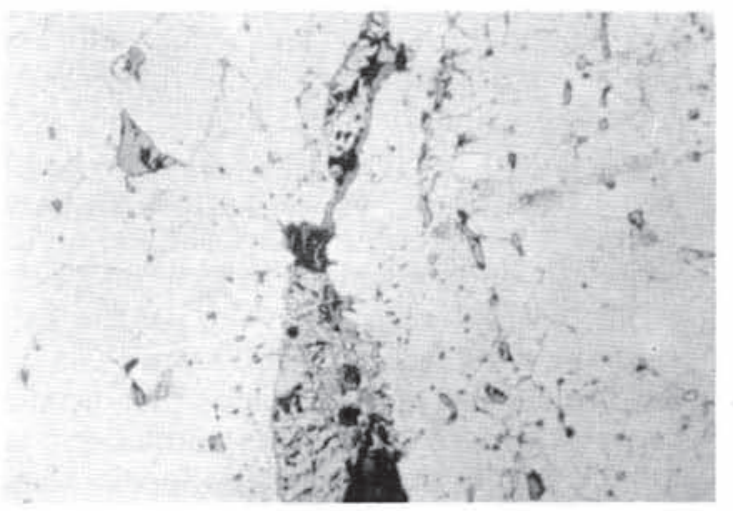

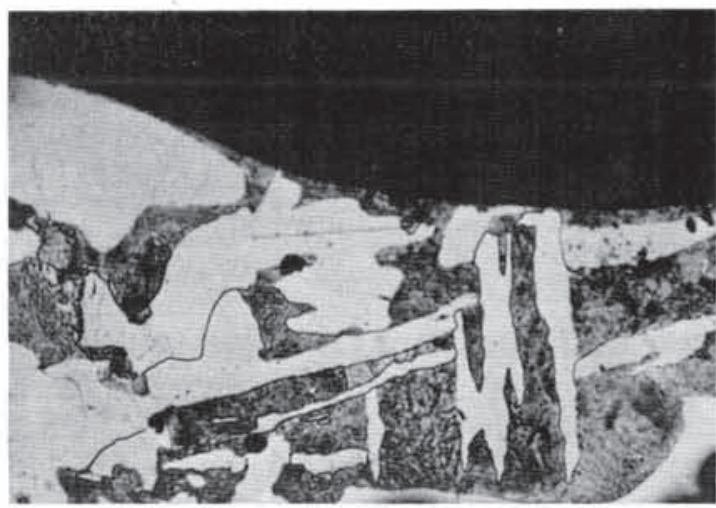

B

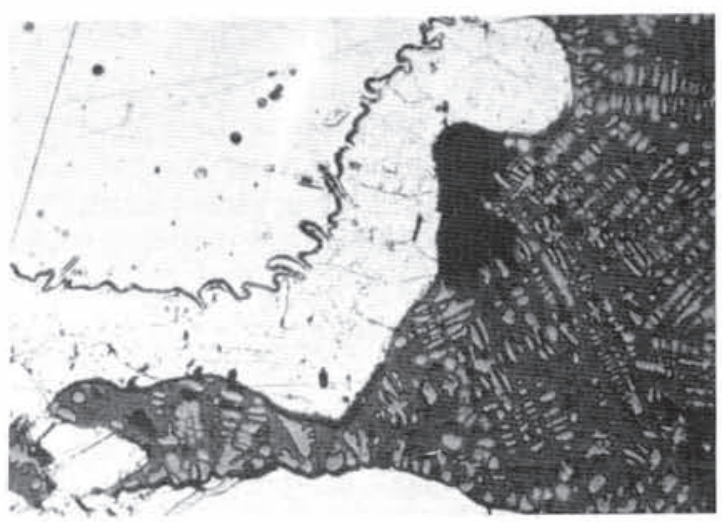

$\mathrm{D}$

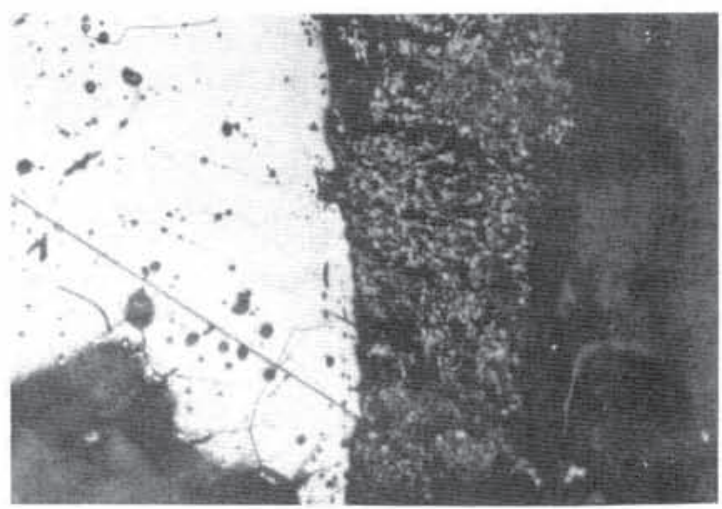

F

Lám. II. Metalografías de las piezas de hierro. A) Hierro dulce (100 X), matriz ferrítica con inclusiones de sulfuros "gris paloma" y escorias, zona de crecimiento irregular, lo que da idea de dos procesos de solidificación superpuestos: uno lento y otro más rápido. B) Hierro dulce $(140 \mathrm{X})$, ferrita y perlita formando una estructura Widmanstätten típica. Escoria formada por dendritas de monóxido de hierro en matriz vidriosa, indicativo de un mal rendimiento en la reducción. C) Hierro semiforjado (100 X), matriz ferrítica con escorias. D) Hierro semiforjado (275 X), ferrita con escaso contenido en perlita, formas sinuosas que sugieren un alto contenido en fósforo. E) Barra de hierro forjado $(100 \mathrm{X})$, sulfuros "gris paloma" en una matriz ferrítica, pieza doblada que ha encerrado en su seno algunas impurezas o escorias. F) Barra de hierro forjado (140 X), matriz ferrítica con inclusiones de escorias. Bandas de óxidos de hierro de alta temperatura formados durante la forja. 
tructura de la escoria hace pensar en una gran cantidad de hierro presente en la misma, lo que es indicativo de una reducción bastante pobre. Por otro lado, dio la casualidad de que la muestra se tomó en una zona con mucho hierro, lo que puede dar una falsa impresión de homogeneidad.

Muestra FrII (Lám. II: c y d) hierro reducido ligéramente forjado. Todavía rico en poros y con escoria rellenando dichos poros. La fase de hierro ha cambiado: hay poca o ninguna perlita; la estructura presenta formas sinuosas de ferrita lo que sugiere un coitenido alto en fósforo (por ejemplo, entre un 0.5 y un $1.5 \%$ ). También el contenido en carbón es bajo en esta muestra. El bajo contenido en carbono y el alto en fósforo sugieren que las muestras 2 y 3 vienen de zonas muy diferentes de la masa reducida original (lo que no deja de ser lógico). La escoria presenta dendritas de $\mathrm{FeO}$ en una matriz obscura, vitrosa; no muestran evidencias de trabajo. Aparecen marcas geométricas dejadas por gotas del ácido usado en la preparación de la muestra.

Muestra FrIII (Lám. III: e y f) hierro forjado, casi sin escoria y doblado una vez. Aparecen óxidos de hierro de alta temperatura, formados durante la forja; No hay impurezas, ya que se forman por la oxidación de hierro $\left(\mathrm{FeO}, \mathrm{Fe}_{3} \mathrm{O}_{4}\right.$, $\left.\mathrm{Fe}_{2} \mathrm{O}_{3}\right)$. También aparecen escorias alargadas, no eliminadas por la forja pero sí deformadas según la dirección del martilleo. Parece que se trata de ferrita con niveles alternos de fósforo (0.1 a $1.5 \%$ P).

Hay gran cantidad de sulfuros (gotas de color gris paloma, no solubles en la matriz), cuyo origen puede estar en el carbón vegetal, que hacen que el metal sea quebradizo: es algo indeseable desde puntos de vista modernos.

\section{CONCLUSIONES}

A la vista de las metalografías, se puede concluir que el resultado no ha sido tan bueno como muchos de los hierros históricos. Pongamos por ejemplo las referencias del Dr. McDonnell (1989: 375 y 376$)$ a los hierros ingleses anteriores a la conquista normanda: los herreros tenían al menos cuatro tipos de hierro diferentes (aunque también es verdad que todos los hierros contenían escorias en mayor o menor medida). Los tipos usados eran: hierro ferrítico ( $<0$ '1 de elementos aleados), hierro fosforoso (0’05-0'5\% de $\mathrm{P}$, lo que impide la difusión del C y por tanto la formación de acero, aumenta la dureza de la ferrita y hace más fragil el hierro, lo que pudo considerarse ventajoso en esos tiempos por la dureza) acero (con C como el principal elemento en aleación) y una cuarta categoría formada por la mezcla de esos tipos.

Un caso extremo de calidad lo da Schmidt (1983: 428, fig. 12): se trata de una pieza africana de hierro (un fragmento de posible brazalete) que iguala piezas europeas de los ss. XVIII y XIX. Es verdad que es difícil hacer comparaciones con este caso dada la gran diferencia de resultados y de tecnología, pero permite tener un punto de referencia y afirmar que se podía conseguir buen hierro en la Antigüedad. Probablemente no debió de ser frecuente un hierro tan bueno como el que comenta Schmidt, pero tampoco debió de serlo uno tan malo como el que se consiguió en Moesgaard.

Con todo, también se documenta el extremo opuesto al señalado por Schmidt, como es el caso de algunos hornos checos conteniendo carbón con restos de escoria (Souchopová, 1989: 153), indicando que también en la Prehistoria se dieron operaciones fallidas.

En Polonia (Nosek, 1985: 169) llegaron a la conclusión de que la reducción se realizó en la Antigüedad con corriente inducida sin la ayuda de fuelles, lo que consideran apoyado por el hecho de que los hornos aparecen siempre en zonas alomadas y porque no se han encontrado toberas, que habrían sido fundamentales de haberse usado fuelles. Ahora bien, Jutlandia es plana (aunque es verdad que allí sopla mucho viento, la cuestión no es tan simple, y, en cualquier caso, no soplaba en el lugar en que se trabajó), y en Moesgaard no se usaron toberas, sino que se enfocaron los boquereles de los fuelles hacia los agujeros, dejando una distancia de seguridad (pero también es verdad que se trataba de fuelles de válvula y no fuelles de bolsa). En Checoslovaquia sí que aparecieron las toberas (Souchopová, 1989: 160) y en Africa igual (Schmidt, 1983: 426). En cualquier caso, parece que sigue siendo una cuestión abierta (y que en realidad pudo darse una mezcla de ambos sistemas, que es lo que pasaba en Moesgaard).

En el caso de la experiencia comentada, el gran éxito fue ya simplemente el haber logrado obtener hierro, dejando claro que la habilidad

T. P., $51, \mathrm{n}^{\circ} 1,1994$ 
del artesano era un factor importante de éxito (y, tal vez, también de especialización).

Tal vez debamos pensar que costaba más de lo que creemos disponer de hierro de calidad o bien, que realizaban alguna otra tarea tras la reducción que hoy se nos escapa. Quedan, por el momento, las metalografías presentadas que podrán servir de referencia para investigaciones futuras en nuestro país.

\section{BIBLIOGRAFÍA}

APPEL, L. (1986): "Jernudvindingsanlaeg ved Maglegaard, Oostermarie". Fra Bornholms Museum: 88-92.

arana Castillo, R.; Muñoz Amilibia, A. Ma ; Ramallo Asensio, S. y Ros Sala, Ma M. (eds.) (1993): "Metalurgia en la Península Ibérica durante el Primer Milenio a.C. Estado actual de la Investigación". Universidad de Murcia. Murcia.

Budd, P.; Gale, D.; Pollard, A. M.; Thomas, R. G. y Williams, P. A. (1992): "The early development of metallurgy in the British Isles". Antiquity, 66, 252: 677686.

Cleere, H. (1970): Sussex Archaeological Society. Occasional Paper No 1.

- (1972): "The classification of early iron-smelting furnaces". The Antiquaries Journal, LII-I: 8-23.

FAUST, B. (1986): "Reduction using carbon". Education in Chemistry, Mayo de 1986: 76-78.

Coghlan, H. H. (1956): Prehistoric and Early Iron in the Old World. Pitt Rivers Museum Occasional Papers in Technology, 8.

GiLchrist, J. D. (1989): “Estraction Metallurgy”. Pergamon Press, Oxford.

Hedeager, L. y Kristiansen, K. (1985): Arkaeologi Leksikon. Politikens Forlag. Copenague.

Hernández Vera, J. A. y Murillo Ramos, J. J. (1985): "Aproximación al estudio de la siderurgia celtibérica del Moncayo". Caesaraugusta 61-62: 177-190.

Hvass, L. (1980): “Boonder, Koobmaen og Krigskarle $i$ Jernalderen". Forlaget Sesam. Copenague.

Jensen, J. (1982): "The Prehistory of Denmark". Methuen \& Co. Londres y Nueva York.
Keesmann, I. y Niemayer, H. G. (1989): “Un centro primitivo de la elaboración de hierro en la factoría fenicia de Toscanos". En C. Domergue (coord.) Minería y Metalurgia en la Antiguas Civilizaciones Mediterráneas y Europeas, I. Ministerio de Cultura. Madrid: 99-108.

LinSLEY, S. M. y HeTHERINGTON, R., (1978): "A seventeenth-century blast furnace at Allensford". Journal of the Historical Metallurgical Society, 12:1-11.

MAdRoñero de LA CAL, A. (1989): "Los hierros de la España Prerromana". En C. Domergue (coord.) Minería y Metalurgia en la Antiguas Civilizaciones Mediterráneas y Europeas, I. Ministerio de Cultura. Madrid 109118.

MANKU, G. S. (1983): "Principios de Química Inorgánica". (Traducción de Raimundo Cea Olivares). McGrawHil. Méjico.

MCDONNEll, G. M. (1989): "Iron and its alloys in the fifth to eleventh centuries A.D. in England". World Archaeology, 20-3: 373-382.

NoseK, E. M. (1985): "The Polish smelting experiments in furnaces with slag pits". En P. T. Craddock y M. J. Hughes (eds.): Furnaces and Smelting Technology in Antiquity. British Museum Occassional Paper 48, Trustees of the British Museum, Londres: 165-177.

ROSTOKER, W. y DVORAK, J. (1990): "Wrought irons: distinguishing between processes". Archaeomaterials, 4: 153-166.

Schmidt, P. R. (con la colaboración de D. H. Avery) (1983): "More evidence for an advanced prehistoric iron technology in Africa". Journal of Field Archaeology, 10: 421-434.

SOUCHOPOVÁ, V. y STRÁNSKY, K. (1989): "Experimental iron smelting in the Blansko Region, Czechoslovakia". Archaeomaterials, 3: 149-161.

TYLECOTE, R. F. (1986): "The Prehistory of Metallurgy in the British Isles". The Institute of Metals, Londres.

- (1987): The Early History of Metallurgy in Europe. Longman, Londres y Nueva York.

TYLECOTE, R. F. y MERKEL, F. (1985): "Experimental smelting techniques: achievements and future”. En P. T. Craddock y M. J. Hughes (eds.): Furnaces and Smelting Technology in Antiquity. British Museum Occassional Paper 48. Trustees of the British Museum, Londres: 3-20.

WaldBaum, J. C. (1989): "Copper, iron, tin wood: the start of the Iron Age in the Eastern Mediterranean". Archaeomaterials, 3-2: 111-122. 